Proyecciones Journal of Mathematics Vol. 33, $\mathrm{N}^{\circ}$ 2, pp. 157-174, June 2014.

Universidad Católica del Norte

Antofagasta - Chile

\title{
On triple difference sequences of real numbers in probabilistic normed spaces
}

\author{
Binod Chandra Tripathy \\ Institute of Advanced Study in Science and Technology, India \\ and \\ Rupanjali Goswami \\ Raha Higher Secondary School, Nagaon \\ Received : December 2013. Accepted : January 2014
}

\begin{abstract}
In this paper we define concept of triple $\Delta$-statistical convergent sequences in probabilistic normed space and give some results. Also we introduce the notions of $\Delta$-statistical limit point and $\Delta$-statistical cluster point and investigate their different properties.
\end{abstract}

AMS Subject classification : 40A05, 40A35, 40B05, 40D25.

Keyword and phrases : Triple sequence, t-norm, probabilistic norm; cluster point; difference operator. 


\section{Introduction}

The notion of probabilistic normed space ( $P N$-space) is a generalization of normed linear space. In an ordinary normed linear space norm of vectors are represented by a positive number. But in a $P N$-space, the norm of vectors are represented by probability distribution functions rather than a positive number. The notion of $P N$-spaces was first introduced by Serstnev [25] in 1963. For detailed history, development and applications in different subjects of the notion of probabilistic normed spaces, one may refer to Alotaibi [1], Alsina etal ([2], [3]), Constantin etal [5], Esi [6], Karakus [11], Menger [18], Lafuerza etal ([14], [15]), Lafuerza etal [16], Schweizer and Sklar ([23], [24]), Tripathy etal [34].

As a generalization of ordinary convergence for sequences of real numbers, the notion of statistical convergence was first introduced by Fast [9]. After then it was studied by many researchers like Connor [4], Fridy [10], Karakus [11], Karakus and Demirci [12], Salat [20], Tripathy ([26], [27]), Tripathy and Baruah [28], Tripathy etal [29], Tripathy and Dutta [32], Tripathy and Sarma [33]. Statistical convergence has been studied in abstract spaces such as the fuzzy number space by Esi ([6], [8]), Fast [9]), locally convex spaces by Maddox [17]. Karakus [11] introduced the notion of statistical convergence in $P N$-spaces and followed by Ideal convergence by Tripathy etal [34], in normed linear spaces by Kolk [13]. Karakus and Demirci [12], studied statistical convergence of double sequences in PN-spaces. In recent times Esi and Özdemir [7] introduced generalized $\Delta^{m}$-statistical convergence in $P N$-spaces for single generalized difference sequences. Also sequences of fuzzy numbers has been studied recently by Tripathy and Borgohain [30] and Tripathy and Debnath [31].

The notion of double sequence was initiated by Priengsheim [19]. In this paper we introduce the concept of statistical convergence of triple difference sequence in probabilistic normed spaces and establish some basic properties in $P N$-spaces.

\section{Definitions and Preliminaries}

Definition 2.1. A function $f: R \rightarrow R_{0}^{+}$is called a distribution function if it is non-decreasing and left continuous with $\inf _{t \in R} f(t)=0$ and 
$\sup _{t \in R} f(t)=1$.

Throughout $D$ denotes the set of all distribution functions.

Definition 2.2. A triangular norm or $t$-norm is a binary operation on $[0,1]$ which is continuous, commutative, associative, non-decreasing and has 1 as neutral element, i.e., it is the continuous mapping $*:[0,1] \times[0,1] \rightarrow[0,1]$ such that for all $a, b, c \in[0,1]$

1) $a * 1=a$.

2) $a * b=b * a$.

3) $c * d \geq a * b$ if $c \geq a$ and $d \geq b$.

4) $(a * b) * c=a *(b * c)$.

Example 2.1. Consider the $*$ operation defined by $a * b=\max \{a+b-1,0\}$. Then $*$ is a $t$-norm. Similarly one may consider $a * b=a b, a * b=\min \{a, b\}$ on $[0,1]$ and verify that these are also $t$-norms.

Definition 2.3. A triplet $(X, N, *)$ is called a probabilistic normed space or a $P N$-space if $X$ is a real vector space, $N$ is a mapping from $X$ into $D$ (for $x \in X$ the distribution function $N(x)$ is denoted by $N_{x}$ and $N_{x}(t)$ is the value of $N_{x}$ at $t \in R$ ) and satisfies the following conditions:

$(\mathrm{PN}-1) N_{x}(0)=0$,

$(\mathrm{PN}-2) N_{x}(t)=1$ for all $t>0$ if and only if $x=0$

$(\mathrm{PN}-3) N_{\alpha x}(t)=N_{x}\left(\frac{t}{|\alpha|}\right)$ for all $\alpha \in R-0$

(PN-4) $N_{x+y}(s+t) \geq N_{x}(s) * N_{y}(t)$ for all $x, y \in X$ and $s, t \in R_{0}^{+}$.

Example 2.2. Let $(X,\|\|$.$) be a normed linear space and \mu \in D$ with $\mu(0)=0$ and $\mu \neq h$, where

$$
h(t)= \begin{cases}0, & \text { for all } t \leq 0 \\ 1, & \text { for all } t>0\end{cases}
$$

Define

$$
N_{x}(t)= \begin{cases}h(t), & \text { for } x=0 \\ \mu\left(\frac{t}{\|x\|}\right), & \text { for } x \neq 0,\end{cases}
$$

where $x \in X, t \in R$. Then $(X, N, *)$ is a $P N$-space. We define the functions $\mu$ and $\mu^{\prime}$ on $R$ by 


$$
\mu(x)= \begin{cases}0, & \text { for } x \leq 0 \\ \frac{x}{1+x}, & \text { for } x>0\end{cases}
$$

and

$$
\mu^{\prime}(x)= \begin{cases}0, & \text { for } x \leq 0 \\ \exp \left(\frac{-1}{x}\right), & \text { for } x>0\end{cases}
$$

Then we obtain the following well known $*$ norms

$$
N_{x}(t)= \begin{cases}h(t), & \text { for } x=0 \\ \frac{t}{t+\|x\|}, & \text { for } x \neq 0 .\end{cases}
$$

and

$$
N_{x}^{\prime}(t)= \begin{cases}h(t), & \text { for } x=0 \\ \exp \left(\frac{-\|x\|}{t}\right), & \text { for } x \neq 0\end{cases}
$$

We recall the concepts of convergence and Cauchy sequences for single sequences in a probabilistic normed space.

Definition 2.4. Let $(X, N, *)$ be a $P N$-space. Then a sequence $x=\left\langle x_{k}\right\rangle$ is said to be convergent to $L \in X$ with respect to the probabilistic norm $N$ if for every $\varepsilon>0$ and $\lambda \in(0,1)$, there exists a positive integer $k_{0}$ such that $N_{x_{k}-L}(\varepsilon)>1-\lambda$, whenever $k>k_{0}$. It is denoted by $N-\lim x_{k}=L$ or $x_{k} \stackrel{N}{\rightarrow} L$ as $k \rightarrow \infty$.

Definition 2.5. Let $(X, N, *)$ be a $P N$-space. Then a sequences $x=\left\langle x_{k}\right\rangle$ is said to be Cauchy sequence with respect to the probabilistic norm $N$ if for every $\varepsilon>0$ and $\lambda \in(0,1)$, there exists a positive integer $k_{0}$ such that $N_{x_{k}-x_{l}}(\varepsilon)>1-\lambda$, whenever $k, l>k_{0}$.

Definition 2.6. Let $(X, N, *)$ be a $P N$-space. Then a sequences $x=\left\langle x_{k}\right\rangle$ is said to be bounded in $X$ if there is $r \in R$ such that $N_{x_{k}}(r)>1-\lambda$ where $\lambda \in(0,1)$, we denote by $\ell_{\infty}^{N}$ the spaces of all bounded sequences in $P N$-space.

Definition 2.7. A triple sequence $x=\left\langle x_{n l k}>\right.$ has Pringsheim limit $L$ (denoted by $P-\lim x=L$ ) provided that given $\varepsilon>0$ there exists $n_{0} \in N$ 
such that $\left|x_{n l k}-L\right|<\varepsilon$ whenever $n, l, k>n_{0}$.

Now we introduce the following notions.

Definition 2.8. A subset $K \in N \times N \times N$ is said to have triple asymptotic density $\delta_{3}(K)$ if $\delta_{3}(K)=\lim _{n, l, k \rightarrow \infty} \frac{1}{n l k} \sum_{i_{1}=1}^{n} \sum_{i_{2}=1}^{l} \sum_{i_{3}=1}^{k} \chi_{K}\left(i_{1}, i_{2}, i_{3}\right)$ exists,

where $\chi_{K}$ is the characteristic function of $K$.

Definition 2.9. A real triple sequence $x=<x_{n l k}>$ is said to be $\Delta$ statistically convergent to $L$, provided that for each $\varepsilon>0$. There exists

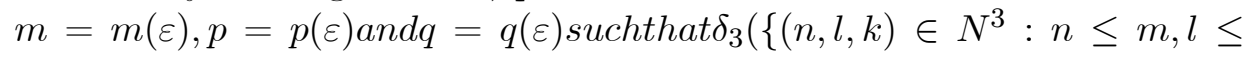
$\left.\left.p, k \leq q,\left|\Delta x_{n l k}-\Delta x_{m} p q\right| \geq \varepsilon\right\}\right)=0$.

Definition 2.10. A real triple sequence $x=\left\langle x_{n l k}\right\rangle$ is said to be $\Delta$ statistically Cauchy, provided that for each $\varepsilon>0$. There exists $m=$ $m(\varepsilon), p=p(\varepsilon)$ and $\mathrm{q}=\mathrm{q}(\varepsilon)$ such that $\delta_{3}\left(\left\{(n, l, k) \in N^{3}: n \leq m, l \leq p, k \leq\right.\right.$ $\left.\left.q,\left|\Delta x_{n l k}-\Delta x_{m p q}\right| \geq \varepsilon\right\}\right)=0$.

Definition 2.11 Let $(X, N, *)$ be a $P N$-space. Then a triple sequences $x=\left\langle x_{n l k}\right\rangle$ is said to be $\Delta$-convergent to $L \in X$ with respect to the probabilistic norm $N$ provided that for every $\varepsilon>0$ and $\lambda \in(0,1)$, there is a positive integer $k_{0}$ such that $N_{\Delta x_{n l k}-L}(\varepsilon)>1-\lambda$ whenever $n \geq k_{0}, l \geq$ $k_{0}, k \geq k_{0}$. In this case we write $N_{\Delta}-\lim x_{n l k}=L$, where $\Delta x_{n l k}=x_{n l k}-$ $x_{n, l+1, k}-x_{n, l, k+1}+x_{n, l+1, k+1}-x_{n+1, l k}+x_{n+1, l+1, k}+x_{n+1, l, k+1}-x_{n+1, l+1, k+1}$ and $\Delta^{0} x_{n l k}=<x_{n l k}>$.

Definition 2.12. Let $(X, N, *)$ be a probabilistic normed space. A triple sequence $x=\left\langle x_{n l k}\right\rangle$ is said to be $\Delta$-Cauchy in $X$ with respect to the probabilistic norm $N$ if for every $\varepsilon>0$ and $\lambda \in(0,1)$, there exists positive integer $k_{0}, k_{1}, k_{2}$ such that $N_{\Delta x_{n l k}-\Delta x_{p q r}}(\varepsilon)>1-\lambda$, whenever $n, p \geq k_{0}$, $l, q \geq k_{1}, k, r \geq k_{2}$.

Definition 2.13. Let $(X, N, *)$ be a probabilistic normed space. A triple sequence $x=\left\langle x_{n l k}>\right.$ is said to be $\Delta$-statistically convergent to $L$ in $X$ with respect to the probabilistic norm $N$ if for every $\varepsilon>0$ and $\lambda \in(0,1)$, $\delta_{3}\left(\left\{(n, l, k) \in N^{3}: N_{\Delta x_{n l k}-L}(\varepsilon) \leq 1-\lambda\right\}\right)=0$. In this case we write $s t_{N \Delta}-\lim x_{n l k}=L$. 
Definition 2.14. Let $(X, N, *)$ be a probabilistic normed space. A triple sequence $x=<x_{n l k}>$ is said to be $\Delta$-statistically Cauchy in $X$ with respect to the probabilistic norm $N$ if for every $\varepsilon>0$ and $\lambda \in(0,1)$, there exist positive integers $N, M$ and $P$ such that $\delta_{3}\left(\left\{(n, l, k) \in N^{3}: N_{\Delta x_{n l k}-\Delta x_{p q r}}(\varepsilon) \leq\right.\right.$ $1-\lambda\})=0$ for all $n, p \geq N, l, q \geq M, k, r \geq N$.

Definition 2.15 Let $(X, N, *)$ be a probabilistic normed space. For $x \in X$, $t>0$ and $0<r<1$, the ball centered at $x$ with radius $r$ is defined by $B(x, r, t)=\left\{y \in X: N_{x-y}(t)>1-r\right\}$.

\section{Main results}

Theorem 3.1. Let $(X, N, *)$ be a $P N$-space, then for every $\varepsilon>0$ and $\lambda \in(0,1)$, the following statements are equivalent.

(i) $s t_{N \Delta}-\lim x_{n l k}=L$.

(ii) $\delta_{3}\left(\left\{(n, l, k) \in N^{3}: N_{\Delta x_{n l k}-L}(\varepsilon) \leq 1-\lambda\right\}\right)=0$.

$\left(\right.$ iii) $\delta_{3}\left(\left\{(n, l, k) \in N^{3}: N_{\Delta x_{n l k}-L}(\varepsilon)>1-\lambda\right\}\right)=1$.

(iv) $s_{\Delta}^{3}-\lim N_{\Delta x_{n l k}-L}(\varepsilon)=1$.

Proof. $(i) \Rightarrow(i i)$ Suppose $s t_{N \Delta}-\lim x_{n l k}=L$. Then by definition we have, for every $\varepsilon>0$ and $\lambda \in(0,1)$, we have $\delta_{3}\left(\left\{(n, l, k) \in N^{3}: N_{\Delta x_{n l k}-L}(\varepsilon) \leq\right.\right.$ $1-\lambda\})=0$.

$(i i) \Rightarrow(i i i)$ Let $\varepsilon>0$ and $\lambda \in(0,1)$, then we have

$\delta_{3}\left(\left\{(n, l, k) \in N^{3}: N_{\Delta x_{n l k}-L}(\varepsilon)>1-\lambda\right\}\right)$

$=1-\delta_{3}\left(\left\{(n, l, k) \in N^{3}: N_{\Delta x_{n l k}-L}(\varepsilon) \leq 1-\lambda\right\}\right)$

$=1$ by $(i i)$.

$($ iii $) \Rightarrow(i v)$ Let $\varepsilon>0$ and $\lambda \in(0,1)$, then we have

$\left\{(n, l, k) \in N^{3}:\left|N_{\Delta x_{n l k}-L}(\varepsilon)-1\right| \geq \lambda\right\}\left\{(n, l, k) \in N^{3}: N_{\Delta x_{n l k}-L}(\varepsilon) \leq\right.$ $1-\lambda\} \cup\left\{(n, l, k) \in N^{3}: N_{\Delta x_{n l k}-L}(\varepsilon) \geq 1+\lambda\right\}$. 
Therefore we have from the finite additivity property of density $\delta_{3}\left(\left\{(n, l, k) \in N^{3}:\left|N_{\Delta x_{n l k}-L}(\varepsilon)-1\right| \geq \lambda\right\}\right)$

$=\delta_{3}\left(\left\{(n, l, k) \in N^{3}: N_{\Delta x_{n l k}-L}(\varepsilon) \leq 1-\lambda\right\}\right)+\delta_{3}\left(\left\{(n, l, k) \in N^{3}:\right.\right.$ $\left.\left.N_{\Delta x_{n l k}-L}(\varepsilon) \geq 1+\lambda\right\}\right)$.

Since, $\delta_{3}\left(\left\{(n, l, k) \in N^{3}: N_{\Delta x_{n l k}-L}(\varepsilon) \leq 1-\lambda\right\}\right)=0$ and $\delta_{3}(\{(n, l, k) \in$ $\left.\left.N^{3}: N_{\Delta x_{n l k}-L}(\varepsilon) \geq 1+\lambda\right\}\right)=0$.

Hence $\delta_{3}\left(\left\{(n, l, k) \in N^{3}:\left|N_{\Delta x_{n l k}-L}(\varepsilon)-1\right| \geq \lambda\right\}\right)=0 \Rightarrow s_{\Delta}^{3}-$ $\lim N_{\Delta x_{n l k}-L}(\varepsilon)=1$.

$(i v) \Rightarrow(i)$ By hypothesis for a given $\varepsilon>0$ and $\lambda \in(0,1)$, we have $\delta_{3}\left(\left\{(n, l, k) \in N^{3}:\left|N_{\Delta x_{n l k}-L}(\varepsilon)-1\right| \geq \lambda\right\}\right)=0$ i.e., $\delta_{3}\left(\left\{(n, l, k) \in N^{3}\right.\right.$ : $\left.\left.N_{\Delta x_{n l k}-L}(\varepsilon) \leq 1-\lambda\right\}\right)+\delta_{3}\left(\left\{(n, l, k) \in N^{3}: N_{\Delta x_{n l k}-L}^{(\varepsilon)-1}+\lambda\right\}\right)=0$.

$\Rightarrow \delta_{3}\left(\left\{(n, l, k) \in N^{3}: N_{\Delta x_{n l k}-L}(\varepsilon) \leq 1-\lambda\right\}\right)=0$, since $\delta_{3}\left(\left\{(n, l, k) \in N^{3}\right.\right.$ : $\left.\left.N_{\Delta x_{n l k}-L}(\varepsilon) \geq 1+\lambda\right\}\right)=0$.

The following result is on the uniqueness of the limit, if it exists.

Theorem 3.2. Let $(X, N, *)$ be a $P N$-space. If a sequence $x=<x_{n l k}>$ is $\Delta$-statistically convergent with respect to the probabilistic norm, then $s t_{N \Delta}-\lim x_{n l k}$ is unique.

Proof. Let $s t_{N \Delta}-\lim x_{n l k}=L_{1}$ and $s t_{N \Delta}-\lim x_{n l k}=L_{2}$, where $x=\left\langle x_{n l k}>\right.$ is a triple sequence. For a given $\lambda>0$ we choose $\gamma \in(0,1)$ such that $(1-\gamma) \star(1-\gamma)>1-\lambda$. Then for any $\varepsilon>0$, we define the following sets.

$$
\begin{aligned}
& K_{N, 1}(\gamma, \varepsilon)=\left\{(n, l, k) \in N^{3}: N_{\Delta x_{n l k}-L_{1}}(\varepsilon) \leq 1-\gamma\right\} \\
& K_{N, 2}(\gamma, \varepsilon)=\left\{(n, l, k) \in N^{3}: N_{\Delta x_{n l k}-L_{2}}(\varepsilon) \leq 1-\gamma\right\}
\end{aligned}
$$

Since $s t_{N \Delta}-\lim x_{n l k}=L_{1}, \delta_{3}\left(\left\{K_{N, 1}(\gamma, \varepsilon)\right\}\right)=0$, for all $\varepsilon>0$.

Furthermore using $s t_{N \Delta}-\lim x_{n l k}=L_{2}$ we get $\delta_{3}\left(\left\{K_{N, 2}(\gamma, \varepsilon)\right\}\right)=$ 0 , for all $\varepsilon>0$. Now let $K_{N}(\gamma, \varepsilon)=K_{N, 1}(\gamma, \varepsilon) \cap K_{N, 2}(\gamma, \varepsilon)$. Then $\delta_{3}\left(\left\{K_{N}(\gamma, \varepsilon)\right\}\right)=0$, which implies that $\delta_{3}\left(\left\{N^{3}-K_{N}(\gamma, \varepsilon)\right\}\right)=1$. If $(n, l, k) \in\left\{N^{3}-K_{N}(\gamma, \varepsilon)\right\}$, then $N_{L_{1}-L_{2}}(\varepsilon) \geq N_{\Delta x_{n l k}-L_{1}}\left(\frac{\varepsilon}{2}\right) \star N_{\Delta x_{n l k}-L_{2}}\left(\frac{\varepsilon}{2}\right)>$ 
$(1-\gamma) \star(1-\gamma)>1-\lambda$. Since $\lambda>0$ is arbitrary we get $N_{L_{1}-L_{2}}(\varepsilon)=1$ for all $\varepsilon>0$, which yields $L_{1}=L_{2}$. Therefore we conclude that $s t_{N \Delta}-$ limit of triple sequence is unique.

Theorem 3.3. Let $(X, N, *)$ be a $P N$-space. If $N_{\Delta}-\lim x_{n l k}=L$, then $s t_{N \Delta}-\lim x_{n l k}=L$, but not necessarily conversely.

proof. By hypothesis $x=\left\langle x_{n l k}>, \Delta\right.$-converges to $L$ with respect to the probabilistic norm $N$. Therefore for every $\lambda>0$ and $\varepsilon>0$ there exists a positive integer $k_{0}$ such that $N_{\Delta x_{n l k}-L}(\varepsilon)>1-\lambda$ for all $n \geq k_{0}, l \geq k_{0}$, $k \geq k_{0}$. Thus the set $\left\{(n, l, k) \in N^{3}: N_{\Delta x_{n l k}-L}(\varepsilon) \leq 1-\lambda\right\}$ has finitely many terms. Since every finite subset of $N^{3}$ has density zero, we see that $\delta_{3}\left(\left\{(n, l, k) \in N^{3}: N_{\Delta x_{n l k}-L}(\varepsilon) \leq 1-\lambda\right\}\right)=0$.

Theorem 3.4. Let $(X, N, *)$ be a $P N$-space and $x=<x_{n l k}>$ be a triple sequence. Then $s t_{N \Delta}-\lim x_{n l k}=L$ if and only if there exists a subset $K=\{(n, l, k): n, l, k=1,2,3,4, \ldots\} \subset N^{3}$ such that $\delta_{3}(K)=1$ and $N_{\Delta}-\lim _{\substack{(n, l, k) \in K \\ n, l, k \rightarrow \infty}} x_{n l k}=L$.

Proof. Suppose $s t_{N \Delta}-\lim x_{n l k}=L$. Now for every $\varepsilon>0$ and $r \in N$, let

$$
M(r, \varepsilon)=\left\{(n, l, k) \in N^{3}: N_{\Delta x_{n l k}-L}(\varepsilon)>1-\frac{1}{r}\right\}
$$

Then $\delta_{3}\{K(r, \varepsilon)\}=0$ and

$$
\begin{gathered}
M(1, \varepsilon) \supset M(2, \varepsilon) \supset M(3, \varepsilon) \supset \ldots \supset M(i, \varepsilon) \supset M(i+1, \varepsilon) \supset \ldots \\
\delta_{3}\{M(r, \varepsilon)\}=1 \text { for } r=1,2,3, \ldots
\end{gathered}
$$

Now we have to show that for $(n, l, k) \in M(r, \varepsilon)$ the sequence $x=x_{n l k}$ is $N_{\Delta}$-convergent to $L$.

Suppose $x=\left\langle x_{n l k}>\right.$ be not $N_{\Delta}$-convergent to $L$. Therefore there exists $\gamma>0$ such that the set $\left\{(n, l, k) \in N_{3}: N_{\Delta x_{n l k}-L}(\varepsilon) \leq 1-\gamma\right\}$ has 
infinitely many terms.

Let $M(\gamma, \varepsilon)=\left\{(n, l, k) \in N^{3}: N_{\Delta x_{n l k}-L}(\varepsilon)>1-\gamma\right\}, \gamma>\frac{1}{r},(r=$ $1,2,3, \ldots)$

Then $\delta_{3}\{M(\gamma, \varepsilon)\}=0$ and by (3.2) we have $M(r, \varepsilon) \subset M(\gamma, \varepsilon)$. Hence $M(r, \varepsilon)=0$ which contradicts (3.3).

Therefore $x=<x_{n l k}>$ is $N_{\Delta}$-convergent to $L$.

Conversely suppose that there exists a subset $K=\{(n, l, k): n, l, k=$ $1,2,3,4, \ldots\} \subset N^{3}$ such that $\delta_{3}(K)=1$ and $N_{\Delta}-\underset{\substack{(n, l, k) \in K \\ n, l, k \rightarrow \infty}}{\lim _{n l k}=L .}$

Then there exists $k_{0} \in N$, such that for every $\gamma \in(0,1)$ and $\varepsilon>0$,

$N_{\Delta x_{n l k}-L}(\varepsilon)>1-\gamma$ for $n \geq k_{0}, l \geq k_{0}, k \geq k_{0}$.

Now, $M(\gamma, \varepsilon)=\left\{(n, l, k) \in N^{3}: N_{\Delta x_{n l k}-L}(\varepsilon) \leq 1-\gamma\right\}$ $\subset N^{3}-\left\{\left(n_{k_{0}+1}, l_{k_{0}+1}, k_{k_{0}+1}\right),\left(n_{k_{0}+2}, l_{k_{0}+2}, k_{k_{0}+2}\right),\left(n_{k_{0}+3}, l_{k_{0}+3}, k_{k_{0}+3}\right), \ldots\right\}$.

Therefore $\delta_{3}(M(\gamma, \varepsilon)) \geq 1-1=0$.

Hence $s t_{N \Delta}-\lim x_{n l k}=L$. This completes the proof.

Theorem 3.5. Let $(X, N, *)$ be a $P N$-space and $x=<x_{n l k}>$ be a sequence whose terms are in the vector space $X$. Then the following conditions are equivalent.

(a) $x$ is $\Delta$-statistically Cauchy sequence with respect to the probabilistic norm $N$.

(b) There exists an increasing index sequence $K=\left\{\left(k_{1}, k_{2}, k_{3}\right)\right\}$ of $N^{3}$ such that $\delta_{3}(K)=1$ and the subsequence $\left\{\left(x_{k_{1}, k_{2}, k_{3}}\right\}_{\left(k_{1}, k_{2}, k_{3}\right) \in K}\right.$ is a $\Delta$ Cauchy sequence with respect to the probabilistic norm $N$.

Theorem 3.6. Let $(X, N, *)$ be a $P N$-space. Then

(i) If $s t_{N \Delta}-\lim x_{n l k}=\xi$ and $s t_{N \Delta}-\lim y_{n l k}=\eta$, then $s t_{N \Delta}-\lim \left(x_{n l k}+\right.$ $\left.y_{n l k}\right)=\xi+\eta$. 
(ii) If $s t_{N \Delta}-\lim x_{n l k}=\xi$ and $\alpha \in R$, then $s t_{N \Delta}-\lim \alpha x_{n l k}=\alpha \xi$.

(iii) If $s t_{N \Delta}-\lim x_{n l k}=\xi$ and $s t_{N \Delta}-\lim y_{n l k}=\eta$, then $s t_{N \Delta}-$ $\lim \left(x_{n l k}-y_{n l k}\right)=\xi-\eta$.

Proof. $(i)$ Let $s t_{N \Delta}-\lim x_{n l k}=\xi$ and $s t_{N \Delta}-\lim y_{n l k}=\eta$. For a given $\varepsilon>0$ and $\lambda \in(0,1)$ we choose $\gamma \in(0,1)$ such that $(1-\gamma) \star(1-\gamma)>$ $1-\lambda$. Then we define the following sets. $K_{N, 1}(\gamma, \varepsilon)=\left\{(n, l, k) \in N^{3}\right.$ : $\left.N_{\Delta x_{n l k}-\xi}(\varepsilon) \leq 1-\gamma\right\}, K_{N, 2}(\gamma, \varepsilon)=\left\{(n, l, k) \in N^{3}: N_{\Delta x_{n l k}-\eta}(\varepsilon) \leq 1-\gamma\right\}$. Since $s t_{N \Delta}-\lim x_{n l k}=\xi, \delta_{3}\left\{K_{N, 1}(\gamma, \varepsilon)\right\}=0$, for all $\varepsilon>0$.

Further using $s t_{N \Delta}-\lim x_{n l k}=\xi$ we get $\delta_{3}\left\{K_{N, 2}(\gamma, \varepsilon)\right\}=0$, for all $\varepsilon>0$.

Let $K_{N}(\gamma, \varepsilon)=K_{N, 1}(\gamma, \varepsilon) \cap K_{N, 2}(\gamma, \varepsilon)$.

Then we observe that $\delta_{3}\left\{K_{N}(\gamma, \varepsilon)\right\}=0$, which implies that $\delta_{3}\left\{N^{3}-\right.$ $\left.K_{N}(\gamma, \varepsilon)\right\}=1$. If $(n, l, k) \in\left\{N^{3}-K_{N}(\gamma, \varepsilon)\right\}$, then we have

$N_{\left(\Delta x_{n l k}-\xi\right)+\left(\Delta y_{n l k}-\eta\right)}(\varepsilon) \geq N_{\Delta x_{n l k}-\xi}\left(\frac{\varepsilon}{2}\right) \star N_{\Delta y_{n l k}-\eta}\left(\frac{\varepsilon}{2}\right)$ $>(1-\gamma) \star(1-\gamma)>1-\lambda$.

This shows that $\delta_{3}\left\{(n, l, k) \in N^{3}: N_{\left.\Delta x_{n l k}-\xi\right)+\left(\Delta y_{n l k}-\eta\right)}(\varepsilon) \leq 1-\lambda=0\right.$.

Hence $s t_{N \Delta}-\lim \left(x_{n l k}+y_{n l k}\right)=\xi+\eta$.

(ii) Let $s t_{N \Delta}-\lim x_{n l k}=\eta, \lambda \in(0,1)$ and $\varepsilon>0$. First we consider the case of $\alpha=0$. In this case, $N_{0 \Delta x_{n l k}-0 \xi}(\varepsilon)=N_{0}(\varepsilon)=1>1-\lambda$.

So we have $N_{\Delta}-\lim 0 x_{n l k}=0$. Then from Theorem 3.2 we have $s t_{N \Delta}-\lim 0 x_{n l k}=0$.

Let $\alpha \in R(\alpha \neq 0)$. Since $s t_{N \Delta}-\lim x_{n l k}=\xi$, we define the following set

$K_{N}(\gamma, \varepsilon)=\left\{(n, l, k) \in N^{3}: N_{\Delta x_{n l k}-\xi}(\varepsilon) \leq 1-\gamma\right\}$, then we can say $\delta_{3}\left\{K_{N}(\gamma, \varepsilon)\right\}=0$ for all $\varepsilon>0$. In this case $\delta_{3}\left\{N^{3}-K_{N}(\gamma, \varepsilon)\right\}=1$. If $(n, l, k) \in N^{3}-K_{N}(\gamma, \varepsilon)$, then

\footnotetext{
$N_{\alpha \Delta x_{n l k}-\alpha \xi}(\varepsilon)=N_{\Delta x_{n l k}-\xi}\left(\frac{\varepsilon}{|\alpha|}\right)$

$\geq N_{\Delta x_{n l k}-\xi}(\varepsilon) \star N_{0}\left(\frac{\varepsilon}{|\alpha|}-\varepsilon\right)$
} 
$=N_{\Delta x_{n l k}-\xi}(\varepsilon) \star 1$

$=N_{\Delta x_{n l k}-\xi}(\varepsilon)>1-\lambda, \alpha \in R(\alpha \neq 0)$

This shows that $\delta_{3}\left\{(n, l, k) \in N^{3}: N_{\alpha \Delta x_{n l k}-\alpha \xi}(\varepsilon) \leq 1-\lambda\right\}=0$

Hence $s t_{N \Delta}-\lim \alpha x_{n l k}=\alpha \xi$.

(iii) From (i) and (ii) by putting $\alpha=-1$, one can get ( $i i i)$.

\section{Statistical limit point and statistical cluster point of the class of difference triple sequences with respect to the probabilistic norm}

Definition 4.1. Let $(X, N, *)$ be a $P N$-space. A subset $Y$ of $X$ is said to be bounded if for every $r \in(0,1)$, there exists $t_{0}>0$ such that $N_{x}\left(t_{0}\right)>1-r$ for all $x \in Y$.

Definition 4.2. Let $(X, N, *)$ be a $P N$-space, then $L \in X$ is called a $\Delta$-limit point of the triple sequence $x=\left\langle x_{n l k}\right\rangle$ with respect to the probabilistic norm $N$ provided that there is a subsequence of $x$ that $\Delta$-converges to $L$ with respect to the probabilistic norm $N$. Let $\Omega_{N \Delta}(x)$ denote the set of all limit points of the sequence $x$. Let $\left\{\left(x_{n\left(i_{1}\right) l\left(i_{2}\right) k\left(i_{3}\right)}\right)\right\}$ be a subsequence of $x=<x_{n l k}>$ and $K=\left\{\left(n\left(i_{1}\right), l\left(i_{2}\right), k\left(i_{3}\right)\right) \in N^{3}, i_{1}, i_{2}, i_{3} \in N\right\}$, then we abbreviate $\left\{\left(x_{n\left(i_{1}\right) l\left(i_{2}\right) k\left(i_{3}\right)}\right)\right\}$ by $\{x\}_{K}$, which in case $\delta_{3}(K)=0,\{x\}_{K}$ is called a subsequence of density zero or thin subsequence. On the other hand $\{x\}_{K}$ is a non-thin subsequence of $x$ if $K$ does not have density zero.

Definition 4.3. Let $(X, N, *)$ be a $P N$-space. Then $\xi \in X$ is called a $\Delta$ statistical limit point of the triple sequence $x=\left\langle x_{n l k}\right\rangle$ with respect to the probabilistic norm $N$ provided that there is a non-thin subsequence of $x$ that $\Delta$-converges to $\xi \in X$ with respect to the probabilistic norm. In this case we say $\xi$ is an $s t_{N \Delta}$-limit point of sequence $x=\left\langle x_{n l k}\right\rangle$. Throughout $\Lambda_{N \Delta}(x)$ denotes the set of all $s t_{N \Delta}$-limit points of the sequence $x$.

Definition 4.4. Let $(X, N, *)$ be a $P N$-space. Then $\gamma \in X$ is called a $\Delta$-statistical cluster point of the sequence $x=\left\langle x_{n l k}\right\rangle$ with respect to the probabilistic norm $N$ provided that for $\varepsilon>0$ and $\lambda \in(0,1)$, $\lim -\sup \delta_{3}\left(\left\{(n, l, k) \in N^{3}: N_{\Delta x_{n l k}-\gamma}(\varepsilon)>1-\lambda\right\}\right)>0$. In this case we say that $\gamma \in X$ is an $s t_{N \Delta}$-cluster point of the sequence $x=<x_{n l k}>$. 
Throughout $\Gamma_{N \Delta}(x)$ denote the set of all $s t_{N \Delta}$-cluster points of the sequence $x$.

Definition 4.5. A probabilistic normed space $(X, N, *)$ is said to be $\Delta$ complete if every $\Delta$-Cauchy sequence is $\Delta$-convergent in $X$ with respect to the probabilistic norm $N$.

Theorem 4.1. Let $(X, N, *)$ be a $P N$-space, then for any sequence $x=<$ $x_{n l k}>\in X, \Lambda_{N \Delta}(x) \subset \Gamma_{N \Delta}(x)$.

Proof. Let $\xi \in \Lambda_{N \Delta}(x)$, then there is a non-thin subsequence

$\left(x_{n\left(i_{1}\right) l\left(i_{2}\right) k\left(i_{3}\right)}\right)$ of $x=<x_{n l k}>$ that $\Delta$-converges to $\xi$ with respect to the probabilistic norm $N$, i.e.

$\delta_{3}\left\{\left(n\left(i_{1}\right), l\left(i_{2}\right), k\left(i_{3}\right)\right) \in N^{3}: N_{\Delta x_{n\left(i_{1}\right) l\left(i_{2}\right) k\left(i_{3}\right)}-\xi}(\varepsilon)>1-\lambda\right\}=d>0$.

Since

$\left\{(n, l, k) \in N^{3}: N_{\Delta x_{n l k}-\xi}(\varepsilon)>1-\lambda\right\} \supset\left\{\left(n\left(i_{1}\right), l\left(i_{2}\right), k\left(i_{3}\right)\right) \in N^{3}:\right.$ $\left.N_{\Delta x_{n\left(i_{1}\right) l\left(i_{2}\right) k\left(i_{3}\right)}-\xi}(\varepsilon)>1-\lambda\right\}$.

For every $\varepsilon>0$, we have $\left\{(n, l, k) \in N^{3}: N_{\Delta x_{n l k}-\xi}(\varepsilon)>1-\lambda\right\}$ $\supseteq\left\{\left(n\left(i_{1}\right), l\left(i_{2}\right), k\left(i_{3}\right)\right) \in N^{3}: i_{1}, i_{2}, i_{3} \in N\right\}-\left\{\left(n\left(i_{1}\right), l\left(i_{2}\right), k\left(i_{3}\right)\right) \in N^{3}:\right.$ $\left.N_{\Delta x_{n\left(i_{1}\right) l\left(i_{2}\right) k\left(i_{3}\right)}-\xi}(\varepsilon) \leq 1-\lambda\right\}$.

Since $\left(x_{n\left(i_{1}\right) l\left(i_{2}\right) k\left(i_{3}\right)}\right)$ is $\Delta$-convergent to $\xi$ with respect to the probabilistic norm $N$, the set $\left\{\left(n\left(i_{1}\right), l\left(i_{2}\right), k\left(i_{3}\right)\right) \in N^{3}: N_{\Delta x_{n\left(i_{1}\right) l\left(i_{2}\right) k\left(i_{3}\right)}-\xi}(\varepsilon) \leq\right.$ $1-\lambda\}$ is finite, for any $\varepsilon>0$, therefore

$$
\begin{aligned}
& \lim \sup \delta_{3}\left(\left\{(n, l, k) \in N^{3}: N_{\Delta x_{n l k}-\xi}(\varepsilon)>1-\lambda\right\}\right) \\
& \geq \limsup \delta_{3}\left\{\left(n\left(i_{1}\right), l\left(i_{2}\right), k\left(i_{3}\right)\right) \in N^{3}: i_{1}, i_{2}, i_{3} \in N\right\} \\
& -\limsup \delta_{3}\left\{\left(n\left(i_{1}\right), l\left(i_{2}\right), k\left(i_{3}\right)\right) \in N^{3}: N_{\Delta x_{n\left(i_{1}\right) l\left(i_{2}\right) k\left(i_{3}\right)}-\xi}(\varepsilon) \leq 1-\lambda\right\} .
\end{aligned}
$$

Hence $\lim \sup \delta_{3}\left(\left\{(n, l, k) \in N^{3}: N_{\Delta x_{n l k}-\xi}(\varepsilon)>1-\lambda\right\}\right)>0$, which implies $\xi \in \Gamma_{N \Delta}(x)$.

Thus $\Lambda_{N \Delta}(x) \subset \Gamma_{N \Delta}(x)$. 
Theorem 4.2. Let $(X, N, *)$ be a $P N$-space. Then for any sequence $x=<x_{n l k}>\in X, \Gamma_{N \Delta}(x) \subset \Omega_{N \Delta}(x)$.

Proof. Let $\gamma \in \Gamma_{N \Delta}(x)$, then $\delta_{3}\left(\left\{(n, l, k) \in N^{3}: N_{\Delta x_{n l k}-\gamma}(\varepsilon)>1-\lambda\right\}\right)>$ 0 for every $\varepsilon>0$ and $\lambda \in(0,1)$. Let $\{x\}_{K}$ be a non-thin subsequence of $x$ such that $K=\left\{\left(n\left(i_{1}\right), l\left(i_{2}\right), k\left(i_{3}\right)\right) \in N^{3}: N_{\Delta x_{n\left(i_{1}\right) l\left(i_{2}\right) k\left(i_{3}\right)}-\gamma}(\varepsilon)>1-\lambda\right\}$ for every $\varepsilon>0$ and $\delta_{3}(K) \neq 0$. Since there are infinitely many elements in $K, \gamma \in \Omega_{N \Delta}(x)$.

Thus $\Gamma_{N \Delta}(x) \subset \Omega_{N \Delta}(x)$.

Theorem 4.3. Let $(X, N, *)$ be a $P N$-space, then for any sequence $x=<$ $x_{n l k}>\in X, s t_{N \Delta}-\lim x_{n l k}=L$, implies $\Lambda_{N \Delta}(x)=\Gamma_{N \Delta}(x)=\{L\}$.

Proof. First we prove that $\Lambda_{N \Delta}(x)=\{L\}$. Suppose that $\Lambda_{N \Delta}(x)=$ $\{L, M\}$ be such that $L \neq M$. In this case, there exist non-thin subsequences $\left\{x_{n\left(i_{1}\right) l\left(i_{2}\right) k\left(i_{3}\right)}\right\}$ and $\left\{x_{p\left(i_{1}\right) q\left(i_{2}\right) r\left(i_{3}\right)}\right\}$ of $x=<x_{n l k}>$ those $\Delta$ converge to $L$ and $M$ respectively with respect to the probabilistic norm $N$. Since $\left\{x_{p\left(i_{1}\right) q\left(i_{2}\right) r\left(i_{3}\right)}\right\}$ is $\Delta$-convergent to $M$ with respect to the probabilistic norm $N$, so for every $\varepsilon>0$ and $\lambda \in(0,1), K=\left\{\left(p\left(i_{1}\right), q\left(i_{2}\right), r\left(i_{3}\right)\right) \in N^{3}\right.$ : $\left.N_{\Delta x_{p\left(i_{1}\right) q\left(i_{2}\right) r\left(i_{3}\right)}-M}(\varepsilon) \leq 1-\lambda\right\}$ is a finite set and so $\delta_{3}(K)=0$.

Then $\left\{\left(p\left(i_{1}\right), q\left(i_{2}\right), r\left(i_{3}\right)\right) \in N^{3}: i_{1}, i_{2}, i_{3} \in N\right\}$

$$
\begin{aligned}
=\left\{\left(p\left(i_{1}\right), q\left(i_{2}\right), r\left(i_{3}\right)\right) \in N^{3}: N_{\Delta x_{p\left(i_{1}\right) q\left(i_{2}\right) r\left(i_{3}\right)}-M}(\varepsilon)>1-\lambda\right\} \\
\quad \cup\left\{\left(p\left(i_{1}\right), q\left(i_{2}\right), r\left(i_{3}\right)\right) \in N^{3}: N_{\Delta x_{p\left(i_{1}\right) q\left(i_{2}\right) r\left(i_{3}\right)}-M}(\varepsilon) \leq 1-\lambda\right\} .
\end{aligned}
$$

Which implies

$$
\delta_{3}\left\{\left(p\left(i_{1}\right), q\left(i_{2}\right), r\left(i_{3}\right)\right) \in N^{3}: N_{\Delta x_{p\left(i_{1}\right) q\left(i_{2}\right) r\left(i_{3}\right)}-M}(\varepsilon)>1-\lambda\right\} \neq 0 .
$$

Since $s t_{N \Delta}-\lim x_{n l k}=L$.

$$
\delta_{3}\left\{(n, l, k) \in N^{3}: N_{\Delta x_{n l k}-L}(\varepsilon) \leq 1-\lambda\right\}=0, \text { for every } \varepsilon>0 .
$$

Therefore we can write $\delta_{3}\left\{(n, l, k) \in N^{3}: N_{\Delta x_{n l k}-L}(\varepsilon)>1-\lambda\right\} \neq 0$. 
For every $L \neq M$, we have

$$
\begin{gathered}
\left\{\left(p\left(i_{1}\right), q\left(i_{2}\right), r\left(i_{3}\right)\right) \in N^{3}: N_{\Delta x_{p\left(i_{1}\right) q\left(i_{2}\right) r\left(i_{3}\right)}-M}(\varepsilon)>1-\lambda\right\} \\
\cap\left\{(n, l, k) \in N^{3}: N_{\Delta x_{n l k}-L}(\varepsilon)>1-\lambda\right\}=\emptyset .
\end{gathered}
$$

Hence $\left\{\left(p\left(i_{1}\right), q\left(i_{2}\right), r\left(i_{3}\right)\right) \in N^{3}: N_{\Delta x_{p\left(i_{1}\right) q\left(i_{2}\right) r\left(i_{3}\right)}-M}(\varepsilon)>1-\lambda\right\} \subseteq$ $\left\{(n, l, k) \in N^{3}: N_{\Delta x_{n l k}-L}(\varepsilon) \leq 1-\lambda\right\}$.

Therefore

$$
\begin{aligned}
& \lim \sup \delta_{3}\left\{\left(p\left(i_{1}\right), q\left(i_{2}\right), r\left(i_{3}\right)\right) \in N^{3}: N_{\Delta x_{p\left(i_{1}\right) q\left(i_{2}\right) r\left(i_{3}\right)}-M}(\varepsilon)>1-\lambda\right\} \\
& \leq \lim \sup \delta_{3}\left\{(n, l, k) \in N^{3}: N_{\Delta x_{n l k}-L}(\varepsilon) \leq 1-\lambda\right\}=0 .
\end{aligned}
$$

This contradicts (4.1).

Hence $\Lambda_{N \Delta}(x)=\{L\}$.

Next we show that $\Gamma_{N \Delta}(x)=\{L\}$. Suppose that $\Gamma_{N \Delta}(x)=\{L, Q\}$ such that $L \neq Q$. Then

$$
\lim \sup \delta_{3}\left\{(n, l, k) \in N^{3}: N_{\Delta x_{n l k}-Q}(\varepsilon)>1-\lambda\right\} \neq 0 .
$$

Since

$\left\{(n, l, k) \in N^{3}: N_{\Delta x_{n l k}-L}(\varepsilon)>1-\lambda\right\} \cap$

$\left\{(n, l, k) \in N^{3}: N_{\Delta x_{n l k}-Q}(\varepsilon)>1-\lambda\right\}=\emptyset$ for every $L \neq Q$, so $\{(n, l, k) \in$ $\left.N^{3}: N_{\Delta x_{n l k}-L}(\varepsilon) \leq 1-\lambda\right\} \supseteq\left\{(n, l, k) \in N^{3}: N_{\Delta x_{n l k}-Q}(\varepsilon)>1-\lambda\right\}$.

\section{Therefore}

$$
\begin{aligned}
& \limsup \delta_{3}\left(\left\{(n, l, k) \in N^{3}: N_{\Delta x_{n l k}-L}(\varepsilon) \leq 1-\lambda\right\}\right) \\
\geq & \lim \sup \delta_{3}\left(\left\{(n, l, k) \in N^{3}: N_{\Delta x_{n l k}-Q}(\varepsilon)>1-\lambda\right\}\right) .
\end{aligned}
$$

From (4.3), the right hand side of (4.4) is greater than zero and from (4.2) the left hand side of (4.4) equals zero. This leads to a contradiction. 
Hence $\Gamma_{N \Delta}(x)=\{L\}$.

Theorem 4.4. Let $(X, N, *)$ be a $P N$-space. Then the set $\Gamma_{N \Delta}$ is closed in $X$ for each $x=<x_{n l k}>$ of elements of $X$.

Proof. Let $y \in \overline{\Gamma_{N \Delta}(x)}$. Let $0<r<1$ and $t>0$, there exists $\gamma \in \Gamma_{N \Delta}(x) \cap B(y, r, t)$ such that $B(y, r, t)=\left\{x \in X: N_{y-x}(t)>1-r\right\}$.

Choose $\eta>0$ such that $B(\gamma, \eta, t) \subset B(y, r, t)$, then we have

$\left\{(n, l, k) \in N^{3}: N_{y-\Delta x_{n l k}}>1-r\right\} \supset\left\{(n, l, k) \in N^{3}: N_{\gamma-\Delta x_{n l k}}(t)>\right.$ $1-\eta\}$.

Since $\gamma \in \Gamma_{N \Delta}(x)$ so $\lim \sup \delta_{3}\left\{(n, l, k) \in N^{3}: N_{\gamma-\Delta x_{n l k}}(t)>1-\eta\right\}>$ 0 .

Hence $\lim \sup \delta_{3}\left\{(n, l, k) \in N^{3}: N_{y-\Delta x_{n l k}}(t)>1-r\right\}>0$.

Thus $y \in \Gamma_{N \Delta}(x)$. This completes the proof.

\section{References}

[1] A. Alotaibi, Generalized statistical convergence in probabilistic normed spaces, The Open Math. Jour.,1, pp. 82-88, (2008).

[2] C. Alsina, B. Schweizer and A. Sklar, On the definition of a probabilistic normed space, Aequat. Math., 46, pp. 91-98, (1993).

[3] C. Alsina, B. Schweizer and A. Sklar, Continuity properties of probabilistic norms, J. Math. Anal. Appl., 208, pp. 446-452, (1997).

[4] J. S. Connor, The statistical and strong $p$-Cesaro convergence of sequences; Analysis, 8, pp. 47-63, (1988).

[5] G. Constantin and I. Istratescu, Elements of Probabilistic Analysis with Applications; vol.36 Mathematics and Its Applications (East European Series), Kluwer Academic Publishers, Dordrecht, Netherlands, (1989). 
[6] A. Esi, The $A$-statistical and strongly $(A-p)$-Cesàro convergence of sequences, Pure Appl. Math. Sci., XLIII(1-2), pp. 89-93, (1996).

[7] A. Esi and M. K. Ozdemir, Generalized $m$-statistical convergence in probabilistic normed space, J. Comput. Anal. Appl., 13(5), pp. 923932, (2011).

[8] A.Esi, Statistical convergence of triple sequences in topological groups, Annals Univ. Craiova, Math. Comput. Sci. Ser., 40(1), pp. 29-33, (2013).

[9] H. Fast, Sur la convergence statistique, Colloq. Math., 2, pp. 241-244, (1995).

[10] J. A. Fridy, On statistical convergence, Analysis, 5, pp. 301-313, (1985).

[11] S. Karakus, Statistical convergence on probabilistic normed space, Math. Commun., 12, pp. 11-23, (2007).

[12] S. Karakus and K. Demirci, Statistical convergence of double sequences on probabilistic normed spaces, Int. J. Math. Math. Sci., (2007), 11 pages, (2007).

[13] E. Kolk, Statistically convergent sequences in normed spaces, Tartu, pp. 63-66, (1988).

[14] B. Lafuerza-Guillen, J. Lallena and C. Sempi, Some classes of probabilistic normed spaces, Rend. Mat. Appl., 17(7), pp. 237-252, (1997).

[15] B. Lafuerza-Guillen, J. Lallena and C. Sempi, A study of boundedness in probabilistic normed spaces, J. Math. Anal. Appl., 232, pp. 183-196, (1999).

[16] B. Lafuerza-Guillen and C. Sempi, Probabilistic norms and convergence of random variables, J. Math. Anal. Appl., 280, pp. 9-16, (2003).

[17] I. J. Maddox, Statistical convergence in a locally convex space, Math. Proc. Camb. Phil. Soc., 104, pp. 141-145, (1988).

[18] K. Menger, Statistical metrics, Proceedings of the National Academy of Sciences of the United States of America, 28(12), pp. 535-537, (1942).

[19] A. Pringsheim, Zur theorie der zweifach unendlichen Zahlenfolgen, Math. Anna., 53, pp. 289-321, (1900). 
[20] T. Salat, On statistically convergent sequences of real numbers, Math. Slovaca, 30, pp. 139-150, (1980).

[21] E. Savas and A. Esi, Statistical convergence of triple sequences on probabilistic normed space, Annals Univ. Craiova, Math. Comput. Sci. Ser., 39(2), pp. 226-236, (2012).

[22] E. Savas and M. Mursaleen, On statistically convergent double sequences of fuzzy numbers, Inform. Sci., 162(3-4), pp. 183-192, (2004).

[23] B. Schweizer and A. Sklar, Probabilistic Metric Spaces, North-Holland Series in Probability and Applied Mathematics, North-Holland, New York, NY,USA, (1983).

[24] B. Schweizer and A. Sklar, Statistical metric spaces, Pacic Jour. Math., 10, pp. 313-334, (1960).

[25] A. N. Serstnev, On the notion of a random normed space, Dokl. Akad. Nauk SSSR, 149, pp. 280-283, (1963).

[26] B. C. Tripathy, Statistically convergent double sequences, Tamkang Jour. Math., 34(3), pp. 231-237, (2003).

[27] B.C. Tripathy, On generalized difference paranormed statistically convergent sequences, Indian J. Pure Appl. Math.,35(5), pp. 655-663, (2004).

[28] B.C. Tripathy and A. Baruah, Lacunary statistically convergent and lacunary strongly convergent generalized difference sequences of fuzzy real numbers, Kyungpook Math. J., 50(4), pp. 565-574, (2010).

[29] B.C. Tripathy, A. Baruah, M.Et and M. Gungor, On almost statistical convergence of new type of generalized difference sequence of fuzzy numbers, Iranian Jour. Sci. Tech., Trans. A : Sci., 36(2), pp. 147-155, (2012).

[30] B.C. Tripathy and S. Borgogain, Some classes of difference sequence spaces of fuzzy real numbers defined by Orlicz function, Advances Fuzzy Syst., 2011, Article ID216414, 6 pages, (2011).

[31] B.C. Tripathy and S. Debnath, On generalized difference sequence spaces of fuzzy numbers, Acta Scientiarum Technology, 35(1), pp. 117$121,(2013)$. 
[32] B.C. Tripathy and H. Dutta, On some lacunary difference sequence spaces defined by a sequence of Orlicz functions and $q$-lacunary $\Delta_{m}^{n}$ statistical convergence, Anal. Stiintifice ale Universitatii Ovidius, Seria Mat., 20(1), pp. 417-430, (2012).

[33] B.C. Tripathy and B. Sarma, Statistically convergent difference double sequence spaces, Acta Math. Sinica(Eng. Ser.), 24(5), pp. 737-742, (2008).

[34] B.C. Tripathy, M. Sen and S. Nath, I-convergence in probabilistic $n$-normed space, Soft Comput., 16, pp. 1021-1027, (2012) DOI 10.1007/s00500-011-0799-8.

\section{Binod Chandra Tripathy}

Mathematical Science Division

Institute of Advanced Study in Science and Technology

Pachim Boragaon, Gorchuk

Guwahati-781035, Assam

India

e-mail : tripathybc@yahoo.com, tripathybc@rediffmail.com

and

\section{Rupanjali Goswami}

Department of Mathematics

Raha Higher Secondary School

Raha, Nagaon-782103, Assam

India

e-mail : rupanjali.goswami@rediffmail.com. 